\title{
Spin injection into a ballistic semiconductor microstructure
}

\section{Citation}

Kravchenko, Vladimir Ya., and Emmanuel I. Rashba. 2003. "Spin Injection into a Ballistic Semiconductor Microstructure." Physical Review B 67 (12) (March 31). doi:10.1103/ physrevb.67.121310.

\section{Published Version}

doi:10.1103/physrevb.67.121310

\section{Permanent link}

http://nrs.harvard.edu/urn-3:HUL.InstRepos:25884368

\section{Terms of Use}

This article was downloaded from Harvard University's DASH repository, and is made available under the terms and conditions applicable to Other Posted Material, as set forth at http:// nrs.harvard.edu/urn-3:HUL.InstRepos:dash.current.terms-of-use\#LAA

\section{Share Your Story}

The Harvard community has made this article openly available.

Please share how this access benefits you. Submit a story.

\section{Accessibility}




\title{
Spin injection into a ballistic semiconductor microstructure
}

\author{
Vladimir Ya. Kravchenko ${ }^{1,4}$ and Emmanuel I. Rashba ${ }^{2,3, *}$ \\ ${ }^{1}$ Argonne National Laboratory, 9700 South Cass Avenue, Argonne, Illinois 60439 \\ ${ }^{2}$ Department of Physics, SUNY at Buffalo, Buffalo, New York 14260 \\ ${ }^{3}$ Department of Physics, MIT, Cambridge, Massachusetts 02139 \\ ${ }^{4}$ Institute of Solid State Physics, Chernogolovka, Moscow District 142432, Russia
}

(Received 9 September 2002; revised manuscript received 7 February 2003; published 31 March 2003)

\begin{abstract}
A theory of spin injection across a ballistic semiconductor embedded between two ferromagnetic leads is developed for the Boltzmann regime. Spin injection coefficient $\gamma$ is suppressed by the Sharvin resistance of the semiconductor $r_{N}^{*}=\left(h / e^{2}\right)\left(\pi^{2} / S_{N}\right)$, where $S_{N}$ is the Fermi-surface cross section. It competes with the diffusion resistances of the ferromagnets $r_{F}$, and $\gamma$ is small, $\gamma \sim r_{F} / r_{N}^{*} \ll 1$, in the absence of contact barriers. Efficient spin injection can be ensured by contact barriers. Explicit formulas for the junction resistance and the spin-valve effect are presented.
\end{abstract}

\section{DOI: 10.1103/PhysRevB.67.121310}

Efficient spin injection from ferromagnetic metals into semiconductors is one of the prerequisites for developing spintronics of the hybrid metal-semiconductor devices. ${ }^{1}$ As distinct from the spin injection into paramagnetic metals, ${ }^{2}$ the first attempts in achieving spin injection into semiconductors failed. In the framework of the diffusion theory, this failure was explained by Schmidt et al. in terms of the "conductivity mismatch," 3 the spin injection coefficient $\gamma$ being of the order of magnitude $\gamma \sim r_{F} / r_{N}$ where $r_{F}$ and $r_{N}$ are the diffusive resistances of a ferromagnet and of a normal conductor (semiconductor microstructure), respectively. Resistive spin-selective contacts had been proposed to remedy the problem, ${ }^{4}$ and an impressive progress in the experimental work was achieved during the last year. ${ }^{5}$ The later theoretical work performed in the framework of the diffusion approximation also substantiated this approach. ${ }^{6-8}$

The spin transistor by Datta and $\operatorname{Das}^{9}$ and similar devices ${ }^{10}$ rely on a ballistic rather than diffusive transport across the semiconductor. Therefore, a lot of theoretical work was performed on a coherent ballistic transport through contacts and a microstructure confined between them. The role of the barriers at the interfaces, the Fresnel-type relations between propagating and reflected waves originating due to the parameter mismatch, and the interference pattern in the bulk caused by the spin-orbit interaction were considered. ${ }^{11}$ The critical role of a barrier at the interface was emphasized $^{12}$ and the scattering in the bulk was discussed. ${ }^{13}$ Spin filtering through perfectly matched interfaces was investigated. $^{14}$

In the theory of spin injection into semiconductors, there exists a gap between the pictures of a diffusive transport and of a coherent transport across the interface. To close this gap, we consider an intermediate regime when (i) the phase coherence at the interfaces is broken and (ii) electrons can be described by the Boltzmann equation inside the microstructure. Solving this equation in a nearly ballistic regime permitted us to find explicit expressions for the basic parameters of the ferromagnet-semiconductor-ferromagnet system. The importance of the problem is emphasized by recent experimental findings of Ramsteiner et al. who investigated spin injection through a high quality $\mathrm{MnAs} / \mathrm{GaAs}$ interface. ${ }^{15}$ They looked for the effect of the symmetry matching of the
PACS number(s): 72.25.Dc, 72.25.Hg

wave functions of MnAs and GaAs and found no correlation between the spin injection and the azimuthal orientation of the surface layers. Apparently, this result reflects the limitations of the current technologies.

We show that the spin injection into a ballistic semiconductor through a diffusive interface is controlled by the Sharvin resistance ${ }^{16}$ of the semiconductor $r_{N}^{*}$ that solely depends on the electron concentration and the resistance quantum $h / e^{2}$. In the absence of the contact resistance, the spin injection coefficient $\gamma \sim r_{F} / r_{N}^{*} \ll 1$. Therefore, contact resistances are indispensable for supporting spin injection from a metal into a semiconductor, similar to the diffusion regime but with a different criterion and because of somewhat different arguments.

Model. We consider a normal ( $N$, nonferromagnetic) conductor residing in a region $-d / 2<x<d / 2$ and separated by barriers from two semi-infinite ferromagnetic $(F)$ leads, $|x|$ $>d / 2$. Spin-orbit interaction is neglected, all conductors are assumed degenerate, and the contacts are both spin selective and spin conserving, i.e., their resistances depend on the electron spin polarization and spin relaxation in the contacts can be disregarded. The scattering at the contacts is diffusive in concord with the assumption of the phase breaking barriers establishing a Boltzmann regime. The specular component of the contact scattering can have no essential effect on the final results because the scattering in ferromagnetic leads is always diffusive. The Boltzmann equation is solved separately in the $N$ and $F$ regions with a proper account of nonequilibrium spins and the boundary conditions at the contacts. We also assume that both contacts and ferromagnets are identical, but the polarization of the ferromagnets may be either parallel (P) or antiparallel (AP), the P and AP geometries in what follows.

$N$ region, $|x|<d / 2$. In the linear approximation in the electrical field $E(x)=-\partial_{x} \varphi(x)$, electrons in the $N$ region can be described by the distribution functions $f_{\alpha}\left(x, v_{x}\right)$ $=f_{0}(\epsilon)+\left(d f_{0} / d \epsilon\right) \psi_{\alpha}\left(x, v_{x}\right)$ where $f_{0}(\epsilon)$ is the Fermi distribution, $\epsilon$ is the energy, $\alpha$ is the spin index, and $\psi_{\alpha}\left(x, v_{x}\right)$ satisfies the Boltzmann equation

$$
v_{x} \partial_{x} \psi_{\alpha}\left(x, v_{x}\right)-e E(x) v_{x}+\left[\psi_{\alpha}\left(x, v_{x}\right)-\bar{\psi}_{\alpha}(x)\right] / \tau_{N}=0 .
$$


Here $\tau_{N}$ is the momentum relaxation time, $\bar{\psi}_{\alpha}(x)$ are the average values of $\psi_{\alpha}\left(x, v_{x}\right)$ over the Fermi sphere, $\bar{\psi}_{\alpha}(x)$ $=\left\langle\psi_{\alpha}\left(x, v_{x}\right)\right\rangle / \rho_{\alpha}, \rho_{\alpha}=\rho_{N} / 2$ are the densities of states of $\alpha$ electrons, and $\rho_{N}$ is the total density of states. Concentrations of nonequilibrium spins are equal to $n_{\alpha}(x)=-\rho_{N} \bar{\psi}_{\alpha}(x) / 2$. Spin relaxation is disregarded.

It is convenient to eliminate the electrical potential $\varphi(x)$ by introducing new functions $e \zeta_{\alpha}\left(x, v_{x}\right)=-\left[\psi_{\alpha}\left(x, v_{x}\right)\right.$ $+e \varphi(x)]$ and their symmetric parts $e \zeta_{\alpha}(x)=-\left[\bar{\psi}_{\alpha}(x)\right.$ $+e \varphi(x)]$. They obey the equation

$$
\zeta_{\alpha}^{\prime}\left(x, v_{x}\right)+\left[\zeta_{\alpha}\left(x, v_{x}\right)-\zeta_{\alpha}(x)\right] / \tau_{N} v_{x}=0 .
$$

When the function $\zeta_{\alpha}(x)$ changes smoothly, it has a meaning of the electrochemical potential of the electrons with the spin $\alpha$. As in similar problems with several groups of carriers, ${ }^{17}$ one should first consider $\zeta_{\alpha}(x)$ as known functions, find $\zeta_{\alpha}\left(x, v_{x}\right)$, and then impose the self-consistency condition $\zeta_{\alpha}(x)=\left\langle\zeta_{\alpha}\left(x, v_{x}\right)\right\rangle / \rho_{\alpha}$.

Equation (1) should be solved for $v_{x}>0$ and $v_{x}<0$ separately. ${ }^{18,19}$ Because of the symmetry of the problem, the solutions obey the relations $\zeta_{\alpha}\left(x, v_{x}\right)$ $=-\zeta_{\bar{\alpha}}\left(-x,-v_{x}\right), \zeta_{\alpha}(x)=-\zeta_{\bar{\alpha}}(-x)$, where $\bar{\alpha}=\alpha$ in the $\mathrm{P}$ geometry, and $\bar{\alpha}=-\alpha$ in the AP geometry. Hence, it is enough to solve Eq. (1) for $v_{x}<0$. For the diffusive contact scattering, the angular distribution of out going electrons is isotropic, $\zeta_{\alpha}\left(d / 2, v_{x}<0\right)=\zeta_{\alpha}^{<}(N)$. The constants $\zeta_{\alpha}^{<}(N)$ scale the antisymmetric in $v_{x}$ parts of $\zeta_{\alpha}\left(x, v_{x}\right)$, and Eq. (1) allows to relate $\zeta_{\alpha}\left(x, v_{x}\right)$ to $\zeta_{\alpha}(x)$.

For the $\mathrm{P}$ geometry, the self-consistency condition for $\zeta_{\alpha}\left(x, v_{x}\right)$ results in an integral equation for $\zeta_{\alpha}(x)$ :

$$
\begin{aligned}
\zeta_{\alpha}(x)= & \frac{1}{2} \zeta_{\alpha}^{<}(N)\left\{E_{2}\left[(d / 2-x) / l_{N}\right]-E_{2}\left[(d / 2+x) / l_{N}\right]\right\} \\
& +\frac{1}{2 l_{N}} \int_{-d / 2}^{d / 2} d u \zeta_{\alpha}(u) E_{1}\left(\frac{|x-u|}{l_{N}}\right),
\end{aligned}
$$

where functions $E_{n}(\xi)=\int_{1}^{\infty} d t e^{-t \xi} / t^{n},{ }^{18} \quad l_{N}=\tau_{N} v_{N}$ is the mean free path, and $v_{N}$ is the Fermi velocity. Function $E_{1}(\xi)$ is related to the integral exponent function, $E_{1}(\xi)$ $=-\operatorname{Ei}(-\xi), \xi>0$. Equation (2) can be solved in the ballistic regime, $d \ll l_{N}$, in powers of a small parameter $\lambda$ $=\left(d / l_{N}\right) \ln \left(l_{N} / d\right) \ll 1$. Under these conditions, the integral term is small and can be disregarded. The function $E_{2}(\xi)$ $=\exp (-\xi)+\xi \operatorname{Ei}(-\xi)$, and the expansion $\operatorname{Ei}(-\xi) \approx \ln \xi$ $+C$, where $C \approx 0.577$ is the Euler constant, can be employed. ${ }^{20}$ Finally, $\zeta_{\alpha}(N) \equiv \zeta_{\alpha}(x=d / 2)$ equals $\zeta_{\alpha}(N)$ $=\zeta_{\alpha}^{<}(N)\left(d / 2 l_{N}\right)\left[1-C+\ln \left(l_{N} / d\right)\right]$. Therefore, the antisymmetric parts of the distribution functions, $\zeta_{\alpha}^{<}(N)$, are large compared to their symmetric parts, $\zeta_{\alpha}(N)$, which are related to the electron concentrations $n_{\alpha}(x)$.

In a similar way, equations for spin-polarized currents $j_{\alpha}$ can be derived. In the leading order in $\lambda$, we find $j_{\alpha}$ $=e^{2} \rho_{N} v_{N} \zeta_{\alpha}^{<}(N) / 4$. Expressing the currents $j_{\alpha}(N)$ through the total current $J$ and the spin injection coefficient $\gamma_{N}$ $=\left[j_{\uparrow}(N)-j_{\downarrow}(N)\right] / J$ results in the final equation

$$
\zeta_{\uparrow}^{<}(N)-\zeta_{\downarrow}^{<}(N)=2 r_{N}^{*} \gamma_{N} J, \quad r_{N}^{*}=2 / e^{2} \rho_{N} v_{N} .
$$

Therefore, in the ballistic region the currents $j_{\alpha}$ and the spin injection coefficient $\gamma_{N}$ are completely determined by the "large" (antisymmetric) parts of the distribution functions. The potential drop inside this region is related to the "small" (symmetric) parts of $\zeta_{\alpha}\left(x, v_{x}\right)$, hence, it is small in $d / l_{N}$ $\ll 1$ and can be disregarded. ${ }^{21}$

We show below that $r_{N}^{*}$ plays a role of the effective resistance of each end of the $N$ region (per unit area). It depends neither on $d$ nor on $l_{N}$ but solely on the electron concentration in the $N$ region

$$
r_{N}^{*}=\left(h / e^{2}\right)\left(\pi^{2} / S_{N}\right)=\left(h / e^{2}\right)(1 / 2 \mathcal{N}),
$$

where $h / e^{2}$ is the resistance quantum. Here $S_{N}=\pi k_{N}^{2}$ is the Fermi-surface cross section, and $\mathcal{N}=k_{N}^{2} / 2 \pi$ is the number of channels per unit cross-section area, including the spin degeneracy factor. The resistance of a narrow diffusive region of the same width $d$ equals $d / \sigma_{N}, \sigma_{N}$ being the conductivity of the $N$ region. It matches $r_{N}^{*}$ smoothly at $d \sim l_{N}$. Because $r_{N}^{*}$ depends on the carrier concentration only, develops due to the electron exchange with the diffusive regions, and has the appropriate analytical form, we identify it as the Sharvin resistance of the normal conductor. ${ }^{16}$ The relevance of Sharvin resistance to the perpendicular transport in layered magnetic structures was recognized by Bauer. ${ }^{22}$

Right $F$ region, $x>d / 2$. The problem of the spin injection into a semi-infinite ferromagnet can be solved when the electron mean free paths $l_{\alpha}$ for both spins are small compared to the spin-diffusion length $L_{F}, l_{\alpha} \ll L_{F}$. Then in a narrow layer near the contact, $w=x-d / 2 \ll L_{F}$, spin relaxation can be neglected, and the self-consistency condition results in the equation for the electrochemical potentials $\zeta_{\alpha}(w)$ similar to Eq. (2),

$$
\begin{aligned}
& \zeta_{\alpha}(w)-\frac{1}{2} \zeta_{\alpha}^{>}(F) E_{2}\left(w / l_{\alpha}\right) \\
& \quad=\frac{1}{2 l_{\alpha}} \int_{0}^{\infty} d u \zeta_{\alpha}(u) E_{1}\left(|u-w| / l_{\alpha}\right) .
\end{aligned}
$$

Here $\zeta_{\alpha}^{>}(F)$ are integration constants similar to $\zeta_{\alpha}^{<}(N)$ but for the right-moving electrons. Eliminating the second term in the left-hand side of Eq. (5) by the shift $\zeta_{\alpha}(x)=\eta_{\alpha}(x)$ $+\zeta_{\alpha}^{>}(F)$ results in a Milne equation ${ }^{18}$

$$
\eta_{\alpha}(w)=\frac{1}{2 l_{\alpha}} \int_{0}^{\infty} d u E_{1}\left(|w-u| / l_{\alpha}\right) \eta_{\alpha}(u) .
$$

For $w \gg l_{\alpha}$, diffusion equations can be used instead of the Milne equations, and the spin relaxation can be easily taken into account. In the region $l_{\alpha} \ll w \ll L_{F}$, both Eqs. (6) and the diffusion equations hold. Therefore, they should match smoothly. For $w \gg l_{\alpha}$, the asymptotic form of the solution of Eq. (6) is

$$
\zeta_{\alpha}(x) \approx \sqrt{3} \eta_{\alpha}(0)\left(w / l_{\alpha}+q_{\infty}\right)+\zeta_{\alpha}^{>}(F), \quad q_{\infty} \approx 0.71 .
$$

The solutions of the standard diffusion equations are ${ }^{8}$

$$
\zeta_{\uparrow}(w)=\left(\sigma_{\downarrow} / \sigma_{F}\right) \zeta_{F} \exp \left(-w / L_{F}\right)+J w / \sigma_{F}+z_{R},
$$




$$
\zeta_{\downarrow}(w)=-\left(\sigma_{\uparrow} / \sigma_{F}\right) \zeta_{F} \exp \left(-w / L_{F}\right)+J w / \sigma_{F}+z_{R},
$$

where $\zeta_{F}$ and $z_{R}$ are integration constants, $\sigma_{\alpha}=e^{2} v_{\alpha} \rho_{\alpha} l_{\alpha} / 3$ are the conductivities for both spins, $v_{\alpha}$ and $\rho_{\alpha}$ are their Fermi velocities and densities of states, and $\sigma_{F}=\sigma_{\uparrow}+\sigma_{\downarrow}$. Matching the small-w expansions of Eqs. (8), $w \ll L_{F}$, with Eq. (7) results in

$$
\zeta_{F}=\sqrt{3} q_{\infty}\left[\eta_{\uparrow}(0)-\eta_{\downarrow}(0)\right]+\left[\zeta_{\uparrow}^{>}(F)-\zeta_{\downarrow}^{>}(F)\right],
$$

where $\quad \sqrt{3} \eta_{\uparrow}(0) / l_{\uparrow}=J / \sigma_{F}-\left(\sigma_{\downarrow} / \sigma_{F}\right)\left(\zeta_{F} / L_{F}\right) \quad$ and $\sqrt{3} \eta_{\downarrow}(0) / l_{\downarrow}=J / \sigma_{F}+\left(\sigma_{\uparrow} / \sigma_{F}\right)\left(\zeta_{F} / L_{F}\right)$. Therefore, $\eta_{\alpha}(0)$ $\sim\left(l_{\alpha} / L_{F}\right) \zeta_{F} \ll \zeta_{F}$ and Eq. (9) reduces to $\zeta_{F}=\zeta_{\uparrow}^{>}(F)$ $-\zeta_{\downarrow}^{>}(F)$. It relates the antisymmetric parts of the distribution functions at the exact boundary of the $F$ region to $\zeta_{F}$ $=\zeta_{\uparrow}(0)-\zeta_{\downarrow}(0)$, i.e., to the difference of the electrochemical potentials of the up- and down-spin electrons near the boundary of the diffusion region.

Spin-polarized currents $j_{\alpha}(F)$ at the boundary of the $F$ region can be found either as $j_{\alpha}(F)=e^{2} v_{\alpha} \rho_{\alpha} \eta_{\alpha}(0) / \sqrt{3}$ or as $j_{\alpha}(F)=\sigma_{\alpha} \partial_{w} \zeta_{\alpha}(w \rightarrow 0)$. Through them the spin injection coefficient $\gamma_{F}$ at the left boundary of the ferromagnet can be found. In the lower order in $l_{\alpha} / L_{F} \ll 1$, the final result is

$$
\gamma_{F}=\Delta \sigma / \sigma_{F}-\left[\zeta_{\uparrow}^{>}(F)-\zeta_{\downarrow}^{>}(F)\right] / 2 r_{F} J,
$$

where $\Delta \sigma=\sigma_{\uparrow}-\sigma_{\downarrow}$, and $r_{F}=\sigma_{F} L_{F} / 4 \sigma_{\uparrow} \sigma_{\downarrow}$ is the effective diffusive resistance of the $F$ region.

Right contact, $x=d / 2$. A tunnel or Schottky barrier separating the $N$ and $F$ regions can be described by the transparency coefficients, $t_{\alpha}^{N F}$ and $t_{\alpha}^{F N}$, for the electrons reaching the contact from its $N$ and $F$ sides, respectively. They are related by the detailed balance condition $\frac{1}{2} t_{\alpha}^{N F} v_{N} \rho_{N}=t_{\alpha}^{F N} v_{\alpha} \rho_{\alpha}$. Spin-polarized currents flowing through a spin-conserving barrier are

$$
j_{\alpha}=-e^{2}\left[t_{\alpha}^{N F}\left\langle\zeta_{\alpha}\left(N, v_{x}\right) v_{x}\right\rangle_{+}+t_{\alpha}^{F N}\left\langle\zeta_{\alpha}\left(F, v_{x}\right) v_{x}\right\rangle_{-}\right]
$$

where the symbols $\langle\cdots\rangle_{ \pm}$indicate that the averaging should be performed only over the right or the left hemisphere, and $\zeta_{\alpha}\left(N, v_{x}\right)$ and $\zeta_{\alpha}\left(F, v_{x}\right)$ are the functions $\zeta_{\alpha}\left(x, v_{x}\right)$ for the incoming electrons at the left and the right side of the contact, respectively. The average values appearing in Eq. (11) can be found from the equations for the currents on both sides of the junction,

$$
\begin{aligned}
& j_{\alpha}(N)=-e^{2}\left[\left\langle\zeta_{\alpha}\left(N, v_{x}\right) v_{x}\right\rangle_{+}+\zeta_{\alpha}^{<}(N)\left\langle v_{x}\right\rangle_{-}\right], \\
& j_{\alpha}(F)=-e^{2}\left[\left\langle\zeta_{\alpha}\left(F, v_{x}\right) v_{x}\right\rangle_{-}+\zeta_{\alpha}^{>}(F)\left\langle v_{x}\right\rangle_{+}\right] .
\end{aligned}
$$

Because of the spin conservation, $j_{\alpha}(N)=j_{\alpha}(F)=j_{\alpha}$, and Eq. (11) can be transformed into the form

$$
j_{\alpha}=\left[\zeta_{\alpha}^{>}(F)-\zeta_{\alpha}^{<}(N)\right] / r_{\alpha},
$$

where the effective contact resistances $r_{\alpha}$ are

$$
r_{\alpha}=4 r_{N}^{*}\left(1-t_{\alpha}^{N F}-t_{\alpha}^{F N}\right) / t_{\alpha}^{N F}
$$

and $r_{N}^{*}$ is defined by Eq. (4).
If the contact behaves like "a two-side black body," i.e., if it absorbs all incoming electrons and ensures an equilibrium emission of them in all directions, then $t_{\alpha}^{F N}+t_{\alpha}^{N F}=1$. This fact follows from arguments similar to those invoked in proving the Kirchhoff theorem in the theory of radiation. Reflection from the barrier and nonequilibrium inside it result in $t_{\alpha}^{F N}+t_{\alpha}^{N F}<1$. Hence, $r_{\alpha}$ are positive and can be considered as contact resistances.

Equation for $\gamma$ following from Eq. (13) is

$$
\gamma_{c}=\Delta r_{c} / r_{c}+\left\{\left[\zeta_{\uparrow}^{>}(F)-\zeta_{\downarrow}^{>}(F)\right]-\left[\zeta_{\uparrow}^{<}(N)-\zeta_{\downarrow}^{<}(N)\right]\right\} / 2 r_{c} J,
$$

where $r_{c}=\left(r_{\uparrow}+r_{\downarrow}\right) / 4, \Delta r_{c}=\left(r_{\downarrow}-r_{\uparrow}\right) / 4$. The denominators 4 ensure direct connection to the diffusive theory.

Spin injection coefficient $\gamma$. Above, transport equations were solved for three different regions of a $F-N-F$ junction: a ballistic $N$ region, diffusive $F$ regions, and resistive contacts. Equations (3), (10), and (15) relate the antisymmetric parts of the distribution functions near the boundaries, $\zeta_{\alpha}^{>}$ and $\zeta_{\alpha}^{<}$, to the spin injection coefficients across them. Remarkably, the final results include exactly the same combinations of the distribution functions despite the drastic difference in the transport mechanisms in the three regions. Therefore, one can eliminate them from Eqs. (3), (10), and (15) and using the spin-current continuity equations, $\gamma_{N}$ $=\gamma_{F}=\gamma_{c} \equiv \gamma$, to derive an equation for $\gamma \cdot{ }^{23}$ Its solution is

$$
\gamma=\left[\Delta r_{c}+r_{F}\left(\Delta \sigma / \sigma_{F}\right)\right] / r_{F N}^{*}
$$

where $r_{F N}^{*}=r_{F}+r_{c}+r_{N}^{*}$. It resembles the result of the diffusive theory for a $F-N-F$ junction: the resistance $r_{F N}^{*}$ in the denominator and spin selectivities of the bulk and the contact in the numerator; cf. Eqs. (36) and (37) of Ref. 8. For a narrow junction, $d \rightarrow 0, r_{N}^{*}$ is standing for $d / 2 \sigma_{N}$. As the total resistance of the junction includes $2 r_{F}$ and $2 r_{c}$, the resistance $r_{N}^{*}$ acquires a meaning of the Sharvin resistance of each end of the ballistic region, the left and the right. It equals $\pi \hbar / e^{2}$ per spin channel. ${ }^{24}$

For the spin injection from a ferromagnetic metal such as Co into a ballistic semiconductor microstructure, $r_{N}^{*}$ $\gtrsim 10^{3} r_{F}$ and with $r_{c} \approx \Delta r_{c} \approx 0$ spin injection is strongly suppressed, $\gamma \sim r_{F} / r_{N}^{*} \ll 1$. In the diffusive regime, a similar effect is attributed to the conductivity mismatch ${ }^{3}$ because of the large diffusive resistance of a semiconductor, $r_{N}$ $=L_{N} / \sigma_{N}, L_{N}$ being the spin-diffusion length in the $N$ conductor. Equation (4) for $r_{N}^{*}$ does not include $\sigma_{N}$ and $L_{N}$, and the large value of $r_{N}^{*}$ comes solely from the low electron concentration, $n_{N}^{(0)} \propto k_{N}^{3}$. However, because the resistances $r_{\alpha}$ are scaled by $r_{N}^{*}$ and enhanced by small $t_{\alpha}^{N F}$, Eq. (14), the spin injection coefficient $\gamma \approx \Delta r_{c} / r_{c}$ may be large enough and is controlled by the contact rather than by the bulk. ${ }^{25} \mathrm{We}$ conclude that $\gamma$ is suppressed even in the ballistic regime and contact resistances are needed to enhance it.

A similar technique can be applied to a contact embedded between semi-infinite $F$ and $N$ regions or to any kind of a ballistic spin filter. ${ }^{26}$ It allows one to calculate $\gamma$ and to relate the contact conductivities $\Sigma_{\alpha}$ of the diffusive theory ${ }^{4,8}$ to the contact resistances $r_{\alpha}$ of Eq. (14). A straightforward calcula- 
tion results in $\Sigma_{\alpha}=1 / r_{\alpha}$. This equation establishes a connection between the parameters of the kinetic and diffusive theories. $^{27}$

$F-N-F$-junction resistance. With the electrochemical potentials $\zeta_{\alpha}(w)$ found above, one can calculate the integration constant $z_{R}$ of Eq. (8). The two-contact resistance of the junction, $R=2 z_{R} / J$, equals

$$
R=-2 \gamma^{2} r_{F N}^{*}+2\left(r_{c}+r_{N}^{*}\right)+2 r_{F}\left(\Delta \sigma / \sigma_{F}\right)^{2} .
$$

Equation (17) differs from the $d \rightarrow 0$ limit of the diffusive theory ${ }^{8}$ by the substitution $r_{c} \rightarrow r_{c}+r_{N}^{*}$.

Similar to the diffusive regime, $R$ can be split into the equilibrium and nonequilibrium parts, ${ }^{4,8} R=R_{\text {eq }}+R_{\text {neq }}$, the latter part turns into zero when $r_{F} \rightarrow 0$ and comes from the nonequilibrium spins in the $F$ regions:

$$
\begin{gathered}
R_{\text {neq }}=2 r_{F}\left[\Delta r_{c}-\left(r_{c}+r_{N}^{*}\right) \Delta \sigma / \sigma_{F}\right]^{2} / r_{F N}^{*}\left(r_{c}+r_{N}^{*}\right), \\
R_{\mathrm{eq}}=2\left(r_{N}^{*}+r_{\uparrow} / 2\right)\left(r_{N}^{*}+r_{\downarrow} / 2\right) /\left(r_{c}+r_{N}^{*}\right) .
\end{gathered}
$$

In the limit $r_{N}^{*}=0$ one recovers the diffusive resistances of Ref. 8 found for $d \rightarrow 0$, cf. Eq. (45) and Appendix.

From Eqs. (16) and (18), a prescription follows: parameters of the ballistic theory can be found from the $d=0$ limit of the diffusive theory by plugging $r_{c} \rightarrow\left(r_{c}+r_{N}^{*}\right)$.

$A P$ geometry and the spin-valve effect. In this geometry $\gamma_{A P}=0$ because of the symmetry arguments. Calculating the resistance shows that $R_{A P}$ differs from $R$ of Eq. (17) only by the absence of the first term. Therefore, the spin-valve effect equals $\Delta R=2 \gamma^{2} r_{F N}^{*}$, where $\gamma$ is determined by Eq. (16).

In conclusion, it is the Sharvin resistance of the ballistic semiconductor microstructure that controls spin injection across a $F-N-F$ junction in the Boltzmann regime. Sharvin resistance is larger than the effective resistances of ferromagnetic leads, and resistive spin-selective contacts are needed to ensure efficient spin injection.

E.I.R. is grateful to Dr. I. Zutic for useful comments. V.Ya.K. acknowledges the support from U.S. DOE Office of Science under Contract No. W31-109-ENG-38, and E.I.R. the support from DARPA/SPINS by the Office of Naval Research Grant No. N000140010819.
*Email address: erashba@mailaps.org

${ }^{1}$ S.A. Wolf et al., Science 294, 1488 (2001); S. Das Sarma et al., Solid State Commun. 119, 207 (2001).

${ }^{2}$ M. Johnson and R.H. Silsbee, Phys. Rev. Lett. 55, 1790 (1985).

${ }^{3}$ G. Schmidt et al., Phys. Rev. B 62, R4790 (2000).

${ }^{4}$ E.I. Rashba, Phys. Rev. B 62, R16 267 (2000).

${ }^{5}$ H.J. Zhu et al., Phys. Rev. Lett. 87, 016601 (2001); A.T. Hanbicki et al., Appl. Phys. Lett. 80, 1240 (2002); S. Kreuzer et al., ibid. 80, 4582 (2002); V.F. Motsnyi et al., ibid. 81, 265 (2002); T. Manago and H. Akinaga, ibid. 81, 694 (2002); P.R. Hammar and M. Johnson, Phys. Rev. Lett. 88, 066806 (2002); C.-M. Hu et al., J. Appl. Phys. 91, 7251 (2002); K.H. Ploog, ibid. 91, 7256 (2002); V. Dediu et al., Solid State Commun. 122, 181 (2002); A. Hirohata et al., Phys. Rev. B 66, 035330 (2002); S.H. Chun et al., ibid. 66, 100408 (2002); P. Van Dorpe et al., cond-mat/0208325 (unpublished).

${ }^{6}$ D.L. Smith and R.N. Silver, Phys. Rev. B 64, 045323 (2001).

${ }^{7}$ A. Fert and H. Jaffrès, Phys. Rev. B 64, 184420 (2001).

${ }^{8}$ E.I. Rashba, Eur. Phys. J. B 29, 513 (2002).

${ }^{9}$ S. Datta and B. Das, Appl. Phys. Lett. 56, 665 (1990).

${ }^{10}$ M. Governale et al., Phys. Rev. B 65, 140403 (2002); R. Ionicioiu and I. D'Amico, Phys. Rev. B 67, 041307 (2003).

${ }^{11}$ H.X. Tang et al., Phys. Rev. B 61, 4437 (2000); L.W. Molenkamp et al., ibid. 64, 121202(R) (2001); T.P. Pareek and P. Bruno, ibid. 65, 241305 (2002); T. Matsuyama et al., ibid. 65, 155322 (2002); M.H. Larsen et al., ibid. 66, 033304 (2002); X.F. Wang et al., Appl. Phys. Lett. 80, 1400 (2002); S.-L. Zhu et al., J. Appl. Phys. 91, 6545 (2002); F. Mireles and G. Kirczenow, Europhys. Lett. 59, 107 (2002).

${ }^{12}$ D. Grundler, Phys. Rev. B 63, 161307(R) (2001); C.-M. Hu and T. Matsuyama, Phys. Rev. Lett. 87, 066803 (2001); H.B. Heersche et al., Phys. Rev. B 64, 161307(R) (2002); T. Schäpers et al., ibid. 64, 125314 (2002).

${ }^{13}$ A. Bournel et al., Eur. Phys. J.: Appl. Phys. 4, 1 (1998); T.P. Pareek and P. Bruno, Phys. Rev. B 65, 241305(R) (2002).

${ }^{14}$ G. Kirczenow, Phys. Rev. B 63, 054422 (2001); P. Mavropoulos et al., ibid. 66, 024416 (2002).

${ }^{15}$ M. Ramsteiner et al., Phys. Rev. B 66, 081304(R) (2002).

${ }^{16}$ Yu.V. Sharvin, Sov. Phys. JETP 21, 655 (1965) [Zh. Eksp. Teor. Fiz. 48, 984 (1965)].

${ }^{17}$ V.Ya. Kravchenko and E.I. Rashba, Sov. Phys. JETP 29, 918 (1969) [Zh. Eksp. Teor. Fiz. 56, 1713 (1969)]; V.Ya. Kravchenko, JETP 94, 603 (2002) [Zh. Eksp. Teor. Fiz. 121, 703 (2002)].

${ }^{18}$ P. M. Morse and H. Feshbach, Methods of Theoretical Physics (McGraw-Hill, New York, 1953), Chap. 12.

${ }^{19}$ K. Fuchs, Proc. Cambridge Philos. Soc. 34, 100 (1938); H. Sondheimer, Adv. Phys. 1, 1 (1952).

${ }^{20}$ I. S. Gradshtein and I. M. Ryzhik, Table of Integrals, Series and Products (Academic, New York, 1980).

${ }^{21}$ Electric field in the $N$ region is small and inhomogeneous, $E(x)$ $\sim\left(r_{N}^{*} J / l_{N}\right) \ln \left[l_{N} /(d / 2-|x|)\right]$.

${ }^{22}$ G.E.W. Bauer, Phys. Rev. Lett. 69, 1676 (1992); Sharvin resistance was also invoked by A.T. Filip et al., Phys. Rev. B 62, 9996 (2000); G. Schmidt and L.W. Molenkamp, Semicond. Sci. Technol. 17, 310 (2002).

${ }^{23}$ This procedure is similar to the $\gamma$ technique (Ref. 8) that turned out to be efficient in the diffusive theory of spin injection.

${ }^{24}$ Sharvin resistances of ferromagnets $r_{\alpha}^{*} \sim\left(h / e^{2}\right) / k_{\alpha}^{2}$, where $k_{\alpha}$ are Fermi momenta, are small as compared to $r_{F}$ in the parameter $l_{\alpha} / L_{F} \ll 1$ and, therefore, can be disregarded.

${ }^{25}$ Similar to the diffusion theory, ${ }^{7,8}$ high contact resistances may modify criteria, e.g., $l_{N} \ll L_{N}$ and $l_{\alpha} \ll L_{F}$. However, the basic conclusion about the role of $r_{N}^{*}$ remains intact.

${ }^{26}$ J.C. Egues, Phys. Rev. Lett. 80, 4578 (1998); T. Koga et al., ibid. 88, 126601 (2002); E.A. de Andrada e Silva and G.C. La Rocca, Phys. Rev. B 59, R15 583 (1999); A. Voskoboynikov et al., ibid. 59, 12514 (1999).

${ }^{27}$ In a different context, effective contact resistance was discussed by B. Laikhtman and S. Luryi, Phys. Rev. B 49, 17177 (1994); J. Barnaś and A. Fert, ibid. 49, 12835 (1994); A. Shpiro and P.M. Levy, ibid. 63, 014419 (2000). 